\title{
Accessing the core of naturalness, nearly degenerate higgsinos, at the LHC
}

\author{
Chengcheng Han, ${ }^{a}$ Doyoun Kim, ${ }^{a}$ Shoaib Munir ${ }^{a}$ and Myeonghun Park ${ }^{a, b, c}$ \\ ${ }^{a}$ Asia Pacific Center for Theoretical Physics, \\ San 31, Hyoja-dong, Nam-gu, Pohang 790-784, Republic of Korea \\ ${ }^{b}$ Department of Physics, \\ Postech, Pohang 790-784, Korea \\ ${ }^{c}$ Kavli IPMU (WPI), The University of Tokyo, \\ Kashiwa, Chiba 277-8583, Japan \\ E-mail: hancheng@apctp.org, doyoun.kim@apctp.org, s.munir@apctp.org, \\ parc@apctp.org
}

AbSTRACT: The presence of two light higgsinos nearly degenerate in mass is one of the important characteristics of supersymmetric models meeting the naturalness criteria. Probing such higgsinos at the LHC is very challenging, in particular when the mass-splitting between them is less than $5 \mathrm{GeV}$. In this study, we analyze such a degenerate higgsino scenario by exploiting the high collinearity between the two muons which originate from the decay of the heavier higgsino into the lighter one and which are accompanied by a high- $p_{T}$ QCD jet. Using our method, we can achieve a statistical significance $\sim 2.9 \sigma$ as well as $S / B \sim 17 \%$ with an integrated luminosity of $3000 \mathrm{fb}^{-1}$ at the $14 \mathrm{TeV}$ LHC, for the pair production of higgsinos with masses $124 \mathrm{GeV}$ and $120 \mathrm{GeV}$. A good sensitivity can be achieved even for a smaller mass-splitting when the higgsinos are lighter.

KEYwords: Supersymmetry Phenomenology, Jets

ARXIV EPRINT: 1502.03734 


\section{Contents}

1 Introduction 1

2 Nearly mass-degenerate higgsinos in the MSSM 3

3 Reconstructing collimated muons 5

3.1 Backgrounds

3.2 Summary of the cuts 8

4 Results of the signal-to-background analysis $\quad 9$

5 Conclusions 11

\section{Introduction}

One of the key theoretical motivations for low-energy supersymmetry (SUSY) is that it provides a framework in which a light Higgs boson can be obtained without invoking unnatural fine-tuning of theory parameters. However, the Higgs boson discovered recently $[1,2]$ at the Large Hadron Collider (LHC) has a mass around $125 \mathrm{GeV}$ and signal rates consistent with those predicted by the Standard Model (SM). These properties of the Higgs boson, in conjunction with the non-observation of supersymmetric particles, have resulted in excluding large portions of the parameter space of the Minimal Supersymmetric Standard Model (MSSM) where the naturalness criteria are satisfied. If the observed Higgs resonance is to be identified with the lightest CP-even Higgs boson, $h$, of the MSSM, TeV-scale SUSY-breaking masses and/or multi-TeV soft trilinear coupling parameters are necessary, so that the Higgs boson mass can be enhanced sufficiently via radiative corrections [3-8]. Furthermore, null results from gluino searches at the LHC Run-I have pushed the lower limit on its mass to the TeV scale [9-12]. While all of this indicates that SUSY lies at the $\mathrm{TeV}$ scale, such a heavy sparticle mass spectrum might spoil the naturalness of the MSSM by requiring excessive fine-tuning for generating the correct Higgs boson mass [13].

In the MSSM, the minimization of the tree-level Higgs potential leads to the following relation between the mass of the $Z$ boson, $m_{Z}$, and the soft SUSY-breaking Higgs sector parameters [14]:

$$
\frac{M_{Z}^{2}}{2}=-\mu^{2}+\frac{m_{H_{d}}^{2}-m_{H_{u}}^{2} \tan ^{2} \beta}{\tan ^{2} \beta-1} \approx-\mu^{2}-m_{H_{u}}^{2} .
$$

The last approximation in the above equation assumes $\tan \beta \gtrsim 10$, where $\tan \beta \equiv v_{u} / v_{d}$, with $v_{u}$ being the vacuum expectation value $(\mathrm{VeV})$ of the $u$-type Higgs doublet and $v_{d}$ that of the $d$-type one. $m_{H_{u}}$ and $m_{H_{d}}$ are the soft SUSY-breaking masses of these two 
Higgs doublets and the parameter $\mu$ is the common mass paramater for the two Higgs superfields, originating in the superpotential of the MSSM. In order to avoid a large finetuning in eq. (1.1), $\mu$ and $m_{H_{u}}$ ought to lie in the $\sim 100 \mathrm{GeV}-200 \mathrm{GeV}$ range.

The MSSM contains four neutralinos, $\tilde{\chi}_{1-4}^{0}$, which are the mass eigenstates resulting from the mixing of the fermion components of the Higgs superfields, known as the higgsi$\operatorname{nos}\left(\widetilde{H}_{d}^{0}, \widetilde{H}_{u}^{0}\right)$, with those of the gauge superfields, the gauginos $\left(\widetilde{B}^{0}, \widetilde{W}^{0}\right)$. The lightest of these neutralinos is a dark matter (DM) candidate when $R$-parity is conserved. The physical masses of these neutralinos are dependent on the soft SUSY-breaking gaugino mass parameters, $M_{1,2}$, as well as the Higgs-higgsino mass parameter $\mu$ mentioned above. The unification of the soft gaugino masses at some very high scale implies that $M_{1}$ and $M_{2}$ are of the same order as the gluino mass parameter $M_{3}$ at the SUSY-breaking scale [15-18]. Thus the exclusion limits on the gluino mass from the LHC, together with the requirement of naturalness, lead to a large splitting between the parameters $M_{1,2}$ and $\mu$. This in turn implies small gaugino-higgsino mixing and, after diagonalization of the neutralino mass matrix, two of the physical neutralinos are gaugino-like while the other two are almost purely higgsinos, which are very close to each other in mass. In fact, for $M_{1}, M_{2} \gtrsim 1.5 \mathrm{TeV}$ and $\mu \sim 150 \mathrm{GeV}$, the mass-splitting, $m_{\tilde{\chi}_{2}^{0}}-m_{\tilde{\chi}_{1}^{0}}$, between the two lightest higgsino-like neutralinos is less than $5 \mathrm{GeV}$. At the same time, the lighter of the two charginos, $\tilde{\chi}_{1}^{ \pm}$, is also a pure higgsino while the heavier, $\tilde{\chi}_{2}^{ \pm}$, a gaugino. In such a scenario, the mass splitting between the lightest chargino and the lightest neutralino, $m_{\tilde{\chi}_{1}^{ \pm}}-m_{\tilde{\chi}_{1}^{0}}$, is typically about half of $m_{\tilde{\chi}_{2}^{0}}-m_{\tilde{\chi}_{1}^{0}}$.

To search for SUSY in the parameter space regions of the MSSM with nearly degenerate higgsinos is one of the major challenges for particle colliders. A lot of emphasis in this regard has been laid on the mono-jet, mono-photon or mono- $Z$ searches at the future experiments [19-36]. However, owing to the very small signal rates as well as the statistical limitations, all these channels are expected to show only percent level yields at the $14 \mathrm{TeV}$ LHC. Several studies [37-51] have suggested that the presence of extra leptons may help in improving the sensitivity for these processes. In [52, 53] such a compressed higgsino spectrum has been probed by tagging an 'opposite sign - same flavor' (OS/SF) lepton pair originating from the decay of a heavy neutralino. However, this method only works well when the higgsino mass-splitting is around $10 \mathrm{GeV}$ or larger. This is for two main reasons. First, for smaller mass-splitting the two leptons produced are too soft to be tagged efficiently. Second, since these leptons are highly collinear, the signal events are diminished by the requirement to isolate them individually.

Therefore, it is imperative to develop new methods for exploring regions of the MSSM parameter space which are consistent with the naturalness criteria [54-62] but which may have stayed hidden at the LHC so far. In this article, we discuss a method for probing the compressed higgsino spectrum in which the two highly collimated muons produced in the decays of $\tilde{\chi}_{2}^{0}$ are identified as a single object. We explain the event selection procedure, specific to the kinematics of our signal process, which can be employed to reduce the backgrounds. Using some benchmark MSSM points consistent with such a scenario, we analyze the sensitivity that can be achieved at the $14 \mathrm{TeV}$ LHC using our method.

The article is organized as follows. In section 2, we briefly discuss the model parameter 


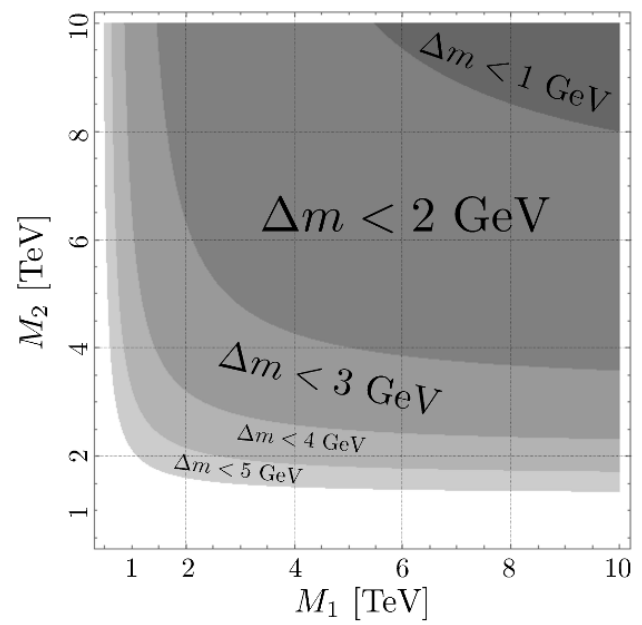

Figure 1. Mass-splitting between the two higgsinos as a function of the gaugino mass parameters $M_{1}$ and $M_{2} \cdot \mu=150 \mathrm{GeV}$ and $\tan \beta=30$.

configurations leading to the scenario of our interest. In section 3 we explain a tagging method for two soft and collimated muons. In section 4, we discuss our numerical results in detail. Finally, we present our conclusions in section 5.

\section{Nearly mass-degenerate higgsinos in the MSSM}

The tree-level neutralino mass matrix in the MSSM is written, in the basis $\left(\widetilde{B}^{0}, \widetilde{W}^{0}, \widetilde{H}_{d}^{0}, \widetilde{H}_{u}^{0}\right)$, as

$$
\mathcal{M}_{\tilde{\chi}^{0}}=\left(\begin{array}{cccc}
M_{1} & 0 & -m_{W} \tan \theta_{W} \cos \beta & m_{W} \tan \theta_{W} \sin \beta \\
& M_{2} & m_{W} \cos \beta & -m_{W} \sin \beta \\
-m_{W} \tan \theta_{W} \cos \beta & m_{W} \cos \beta & 0 & -\mu \\
m_{W} \tan \theta_{W} \sin \beta & -m_{W} \sin \beta & -\mu & 0
\end{array}\right),
$$

where $m_{W}$ is the mass of the $W$ boson and $\theta_{W}$ is the weak mixing angle. The above mass matrix can be diagonalized with an orthogonal real matrix $N$, as $N M_{\tilde{\chi}^{0}} N^{T}=$ $\operatorname{diag}\left(m_{\tilde{\chi}_{1}^{0}}, m_{\tilde{\chi}_{2}^{0}}, m_{\tilde{\chi}_{3}^{0}}, m_{\tilde{\chi}_{4}^{0}}\right)$, such that $m_{\tilde{\chi}_{1}^{0}}<m_{\tilde{\chi}_{2}^{0}}<m_{\tilde{\chi}_{3}^{0}}<m_{\tilde{\chi}_{4}^{0}}$. By assuming $M_{1}, M_{2} \gg$ $|\mu|$ in the above mass matrix, one obtains the approximate relation,

$$
\Delta m \equiv m_{\tilde{\chi}_{2}^{0}}-m_{\tilde{\chi}_{1}^{0}} \approx \frac{m_{W}^{2}}{M_{2}}+\frac{m_{W}^{2} \tan ^{2} \theta_{W}}{M_{1}} .
$$

Similarly, using also the chargino mass matrix, one gets $m_{\tilde{\chi}_{1}^{ \pm}}-m_{\tilde{\chi}_{1}^{0}} \approx \frac{\Delta m}{2}$ (ignoring the terms proportional to $1 / \tan \beta$ ). The neutralino mass matrix in eq. (2.1) is subject to higher order corrections. The diagonalization of the mass matrix in which such corrections have been included (at a certain perturbative order) can be conveniently done numerically using publicly available SUSY mass spectrum calculators. We used the program SUSY-HIT v1.4 [63] to scan over $M_{1}$ and $M_{2}$, both ranging from $0.5 \mathrm{TeV}$ to $10 \mathrm{TeV}$. For this scan we 


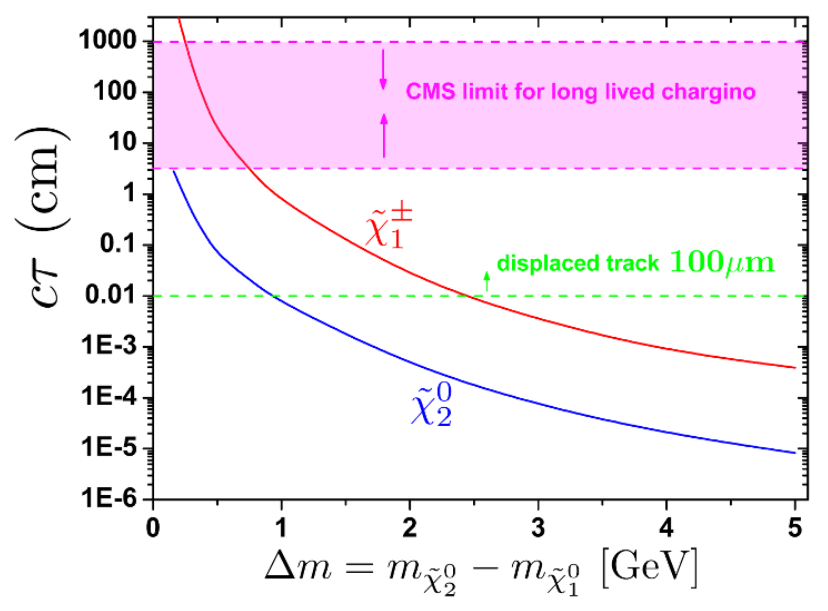

Figure 2. Lifetimes of $\tilde{\chi}_{1}^{ \pm}$and $\tilde{\chi}_{2}^{0}$ as functions of $\Delta m$, which is varied by adjusting $M\left(=M_{1}=M_{2}\right)$. The values of $\mu$ and $\tan \beta$ used are the same as in figure 1 .

set $\mu=150 \mathrm{GeV}, \tan \beta=30$ and the input masses of the SUSY particles other than the electroweakinos to very high values so that they are effectively decoupled. The resultant values of $\Delta m$ are shown in figure 1 . We see that for $M_{1,2} \gtrsim 1.5 \mathrm{TeV}$ the mass-splitting between the higgsino-like $\tilde{\chi}_{1}^{0}$ and $\tilde{\chi}_{2}^{0}$ is always less than $5 \mathrm{GeV}$. Also, for such large $M_{1,2}$, the DM direct detection facility XENON1T [64] will not be sensitive to the $\tilde{\chi}_{1}^{0}[28,34]$, which is the lightest SUSY particle (LSP), when its mass is less than $200 \mathrm{GeV}$.

Since $\tilde{\chi}_{2}^{0}$ is almost mass-degenerate with $\tilde{\chi}_{1}^{0}$, the former can live long enough to leave a secondary vertex in the detector. In figure 2 , we show the lifetime of $\tilde{\chi}_{2}^{0}$, calculated with SUSY-HIT, as a function of $\Delta m$. We see that, for $\Delta m<1 \mathrm{GeV}$, the lifetime of $\tilde{\chi}_{2}^{0}$ can be long enough to produce displaced vertices of order $100 \mu m$. In fact, for $\Delta m<0.1 \mathrm{GeV}$, $\tilde{\chi}_{2}^{0}$ can become collider-stable, so that it leaves the detector before decaying. However, such a tiny higgsino mass-splitting only occurs for $M_{1,2}$ of $\mathcal{O}(100 \mathrm{TeV})$. One also sees in the figure that a chargino has a slightly longer lifetime than a neutralino, which is because $m_{\tilde{\chi}_{1}^{ \pm}}-m_{\tilde{\chi}_{1}^{0}} \approx \frac{\Delta m}{2}$, as noted above. A strong limit on the chargino lifetime, shown by the pink/shaded region in the figure, has recently been obtained by the CMS collaboration [65] for $\Delta m<1 \mathrm{GeV}$. At the LHC, a mono-jet along with a displaced vertex larger than $100 \mu \mathrm{m}$ might help probe the region with $\Delta m<2.5 \mathrm{GeV}$.

A number of studies, as noted earlier, have explored the MSSM regions with $\Delta m>$ $10 \mathrm{GeV}$ in decays of $\tilde{\chi}_{2}^{0}$ that involve two leptons in the final state. Here we will focus on the splitting region $2 \mathrm{GeV} \lesssim \Delta m \lesssim 5 \mathrm{GeV}$ for the decay process

$$
\tilde{\chi}_{2}^{0} \rightarrow \tilde{\chi}_{1}^{0} Z^{*} \rightarrow \tilde{\chi}_{1}^{0} \ell^{+} \ell^{-}
$$

with the $\tilde{\chi}_{2}^{0}$ produced via $p p \rightarrow \tilde{\chi}_{2}^{0} \tilde{\chi}_{1}^{0}+X$. In our case, due to the small mass-splitting between the higgsinos, the two leptons are generally very soft. Therefore, we only consider muons in the final state on account of a much cleaner background as well as a much higher trigger efficiency in their case compared to those for taus or electrons. 


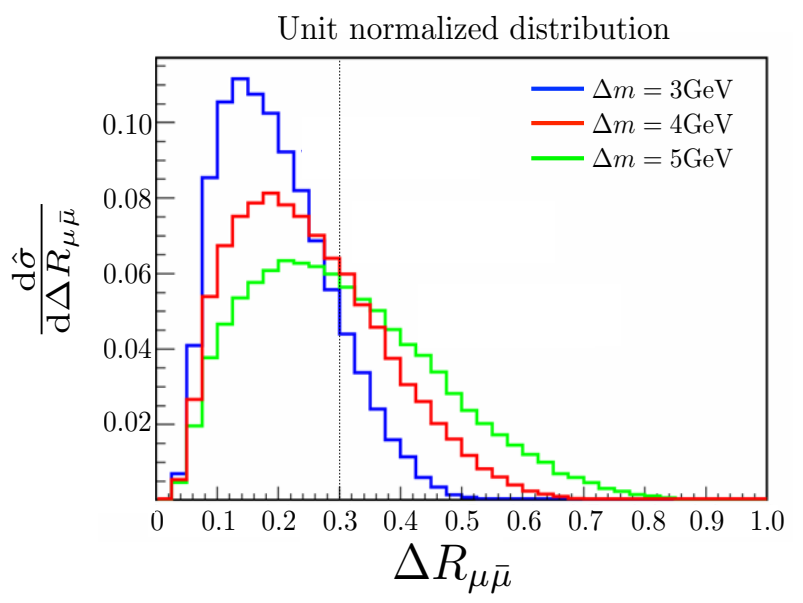

Figure 3. The separation $\Delta R_{\mu \bar{\mu}}$ between the two muons coming from the $Z^{*}$. The dotted vertical line shows that with the conventional cut, $\Delta R^{\max }=0.3$, the muons in the signal process can not be isolated.

In figure 3 we show the separation, $\Delta R_{\mu \bar{\mu}} \equiv \sqrt{\Delta \eta^{2}+\Delta \phi^{2}}$ (with $\eta$ being the pseudorapidity and $\phi$ being the azimuthal angle), between the two final-state muons $\left(p_{T}(\mu)>\right.$ $5 \mathrm{GeV}$ ), for $\Delta m=3,4,5 \mathrm{GeV}$. Evidently, the usual isolation criteria for a single lepton, $\Delta R^{\max }=0.3$, will remove a large number of the signal events. Thus we need to use an unconventional reconstruction method for probing such collimated muons and establishing our signal over the SM backgrounds.

\section{Reconstructing collimated muons}

In order to probe the highly collinear muons produced for a very small $\Delta m$, we cluster them together into one object, $\mu_{\mathrm{col}}$, during our simulation of the higgsino pair-production process. This method is similar in concept to the identification of a 'lepton-jet' [66-71] and has already been used recently in analyses of the decays of a light dark photon or of a light scalar or pseudoscalar $(\leq 3 \mathrm{GeV})[72]$ into two or more soft leptons. Instead of imposing the conventional criterion of $\Delta R^{\max }=0.1$ in order to identify the two muons coming from the $Z^{*}$ as a lepton-jet, we use a modification of the criteria described in the CMS analyses $[72,73]$ for probing collimated muons. Our method is explained below.

- Capturing $\mu_{\mathrm{col}}$ : We require $p_{T}>5 \mathrm{GeV}$ for each muon in the signal, before isolation. In addition to this, we impose the cut $m_{\mu \bar{\mu}}<5 \mathrm{GeV}$ on the invariant mass of the muon pair, since we are only interested in $\Delta m<5 \mathrm{GeV}$.

- Isolation: To suppress the backgrounds containing muon pairs from meson decays, we apply an isolation criterion, $I_{\text {sum }}<3 \mathrm{GeV}$, on $\mu_{\text {col }}$. The isolation parameter $I_{\text {sum }}$ is defined as the scalar sum of the transverse momenta of all additional charged tracks, each with $p_{T}>0.5 \mathrm{GeV}$, within a cone centered along the momentum vector of $\mu_{\text {col }}$ and satisfying $\Delta R^{\max }=0.5$. 


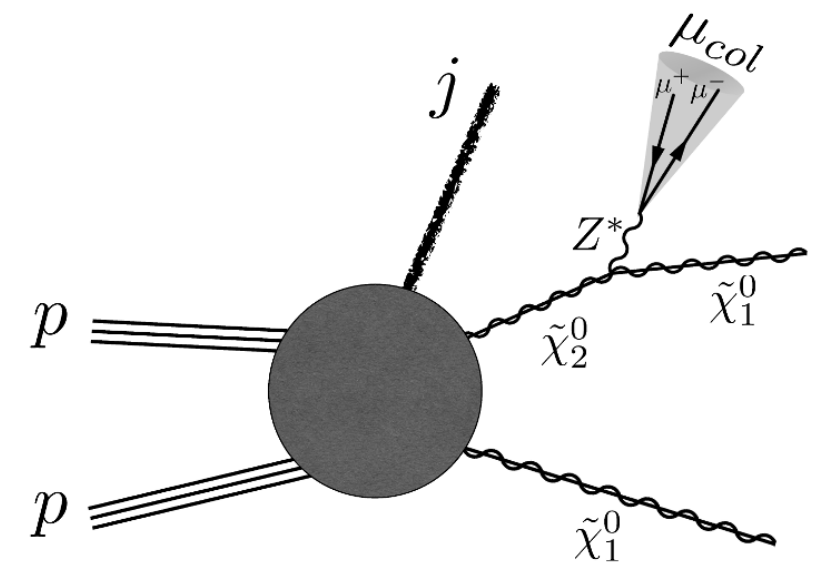

Figure 4. Feynman graph for our signal process. The cone signifies a pair of collimated muons $\mu_{\text {col }}$ coming from the $Z^{*} . j$ denotes a hard QCD jet from initial state radiation.

For the Monte Carlo simulations, we generated the parton-level signal and background events with MadGraph_aMC@NLO [74]. These events were then passed on PythiA 6 [75] for hadronization and subsequently to the fast detector simulator DeLPHES 3 [76] interfaced with FASTJET-v3.0.6 [77] for jet-clustering. In Delphes 3 we added a class for $\mu_{\text {col }}$ identification. The jets were clustered using the anti- $k_{T}[78]$ algorithm with $\Delta R^{\max }$ set to 0.4. As a test of the implementation of our method, we first performed simulations for the benchmark points provided in the CMS analysis [72] and found our results to be within $5 \%$ of the ones presented there, in terms of signal efficiencies.

According to $[72,73]$, the largest background for our signal process, is the $b \bar{b}$ production, which has a cross section of $\mathcal{O}\left(10^{8} \mathrm{pb}\right)$. Although requiring $b$-quarks decays, via double semileptonic decays, into pairs of muons (the branching ratio being around 1\%) which are isolated, reduces this background by $\mathcal{O}\left(10^{-2}\right)$, it is still huge. We therefore apply a parton-level cut of $p_{T}>200 \mathrm{GeV}$ on the first leading jet in the signal and the backgrounds. This translates into the requirement of large missing energy, $\mathbb{E}_{T}$, in the final state at the detector level, which almost entirely removes the $b \bar{b}$ background. To include QCD effects [79], we use the MLM-scheme to match the additional two jets [80]. We illustrate our signal process in figure 4.

In figure 5 we show the combined cross section, after imposing the $p_{T}>200 \mathrm{GeV}$ cut on the leading jet, for our signal process $p p \rightarrow \tilde{\chi}_{2}^{0} \tilde{\chi}_{1}^{0}+X$, and two additional processes, $p p \rightarrow \tilde{\chi}_{2}^{0} \tilde{\chi}_{1}^{ \pm}+X$ and $\rightarrow \tilde{\chi}_{2}^{0} \tilde{\chi}_{2}^{0}+X$, for three different values of $\Delta m$. The reason for including the latter two processes is that the $\tilde{\chi}_{1}^{ \pm}$in the second process as well as the additional $\tilde{\chi}_{2}^{0}$ in the third process gives very soft products which escape undetected, thus resulting in only $\mathbb{E}_{T}$ in the final state and thereby mimicking the signal process. We see in the figure that the cross section gets considerably reduced for smaller mass-splitting. However, even with $\Delta m=3 \mathrm{GeV}$ for a $\sim 200 \mathrm{GeV} \tilde{\chi}_{1}^{0}$, more than 20 signal events can be obtained at the $14 \mathrm{TeV}$ LHC with an integrated luminosity, $\mathcal{L}$, of $3000 \mathrm{fb}^{-1}$. 


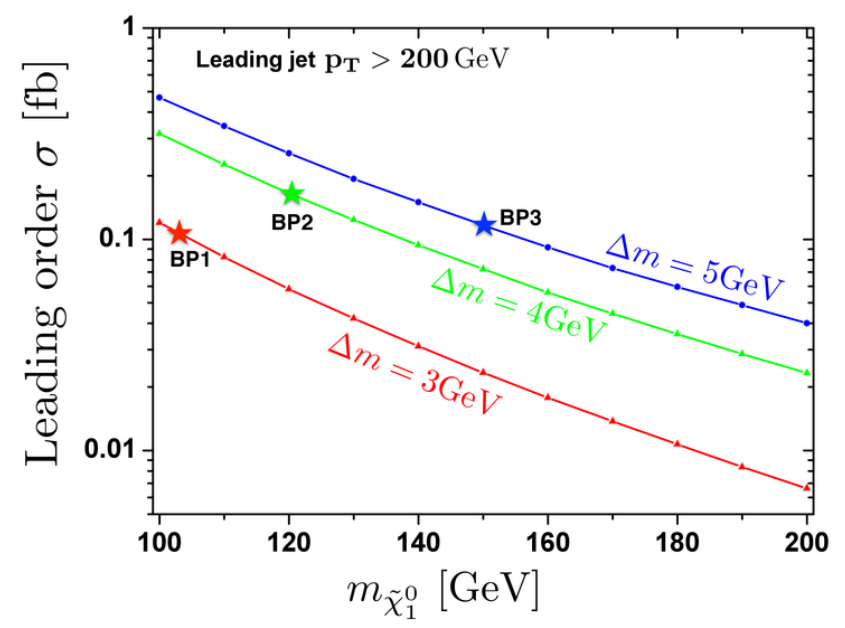

Figure 5. The leading order cross sections corresponding to three different values of $\Delta m$, as a function of the $\tilde{\chi}_{1}^{0}$ mass. We require $p_{T}>200 \mathrm{GeV}$ for the leading jet, $p_{T}>5 \mathrm{GeV}$ for each muon and the detector geometry cuts to be satisfied.

\begin{tabular}{|c|c|c|c|}
\hline & BP1 & BP2 & BP3 \\
\hline$m_{\tilde{\chi}_{1}^{0}}(\mathrm{GeV})$ & 103.0 & 120.0 & 150.0 \\
\hline$\Delta m(\mathrm{GeV})$ & 3.0 & 4.0 & 5.0 \\
\hline Signal cross section $(\mathrm{fb})$ & 0.12 & 0.165 & 0.116 \\
\hline
\end{tabular}

Table 1. Some properties of the three benchmark points analyzed in this study.

For our signal-to-background analysis, we choose three benchmark points (BPs) with a different value of $\Delta m$ each. Some specifics of these points are given in table 1 . The signal cross sections given in the table have been obtained after requiring $p_{T}>200 \mathrm{GeV}$ for the leading jet and $p_{T}>5 \mathrm{GeV}$ for each muon. The detector geometry cuts on the pseudorapidities of the muons $(|\eta|<2.5)$ and the leading jet $(|\eta|<5.0)$ have also been imposed.

\subsection{Backgrounds}

After removing the $b \bar{b}$ background for mono-jet production along with soft muons and $\mathbb{E}_{T}$, the main backgrounds that remain include the following.

- $V+\gamma^{*}+j e t s:$ A large $\mathbb{E}_{T}$ results from the $W \rightarrow \ell \nu$ decay or the $Z \rightarrow \nu \nu$ decay and the two collinear muons originate from the virtual photon, $\gamma^{*}$. To reduce this background, we require $m_{\mu \bar{\mu}}>1.0 \mathrm{GeV}$ and $\Delta R_{\mu \bar{\mu}}>0.1$.

- $\tau \bar{\tau}+j e t s$ : Each tau decays into a muon and a pair of neutrinos. Due to the large boost in the leading jet, these muons become highly collimated. The neutrinos produced are responsible for a large $\mathbb{E}_{T}$. 


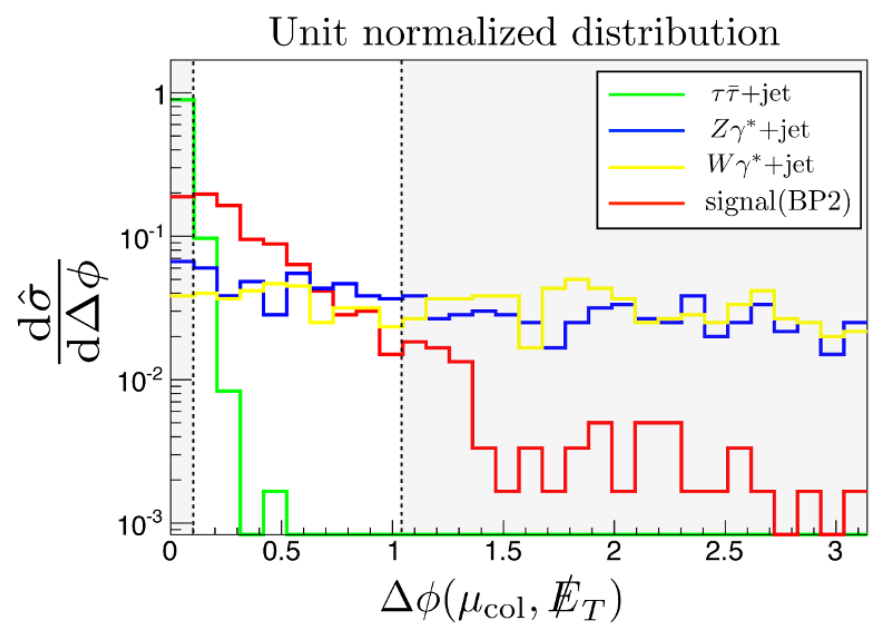

Figure 6. The distribution of $\Delta \phi\left(\mu_{\mathrm{col}}, \mathbb{E}_{T}\right)$ for the signal and the backgrounds. The signal corresponds to our BP2. The grey/shaded regions are the ones cut off in our kinematical analysis.

We also look into some other backgrounds, described below, which are only $\mathcal{O}\left(10^{-2}\right)$ of the above main backgrounds.

- $V b \bar{b}+j e t s$ and $Z b+j e t s$ : This background mimics our signal when the $b$-quarks decay into pairs of muons via double semileptonic decays. However, it is suppressed by a factor $\sim 10^{-4}$ after isolation. We can estimate this background from the ATLAS mono-jet search [81], where a set of mono-jet cuts is imposed on the backgrounds $\left(p_{T}>280 \mathrm{GeV}\right.$ for the leading jet and $\left.\mathbb{E}_{T}>220 \mathrm{GeV}\right)$. The cross section for the $Z(\rightarrow \nu \bar{\nu})+$ jets background is around $0.82 \mathrm{pb}$. The cross section for $W+$ jets, where $W$ decays semileptonically, is around $0.6 \mathrm{pb}$. The total cross section for all these backgrounds thus adds up to about $1.4 \mathrm{pb}$ at the $8 \mathrm{TeV}$ LHC. At the $14 \mathrm{TeV}$ LHC, even if one assumes the cross section for these backgrounds to increase by a factor of 10 , it will reduce to $\sim 0.3 \mathrm{fb}$ after taking into account the suppression of $2 \times 10^{-5}$ from the possibility of the $b$-jets giving collinear muons. Thus this background becomes much smaller than the $V+\gamma^{*}+$ jets backgrounds, which are still around $20 \mathrm{fb}$ after the mono-jet cuts.

The $W(\rightarrow \mu \nu) b \bar{b}+j e t s$ background can also mimic our signal if a $b$-jet is miss-tagged as a muon. The $b$ miss-tagging rate after passing the isolation criteria is less than 0.005 , but since this muon tends to have a large separation from a muon resulting from the $W$ decay, this contribution is also small.

- $t \bar{t}+$ jets: After imposing the mono-jet cut and requiring $m_{\mu \bar{\mu}}<5 \mathrm{GeV}$, the cross section for this background is reduced to less than $0.1 \mathrm{fb}$. We, therefore, do not take it into consideration here.

\subsection{Summary of the cuts}

Below we summarize our cuts based on the discussion above. 
- Mono-jet cut: We require $p_{T}>250 \mathrm{GeV}$ for the leading jet and veto events which have more than three jets with $p_{T}>30 \mathrm{GeV} . \Delta \phi$ between the leading jet and the second jet should be larger than 0.4. All the jets are $b$ - and $\tau$-vetoed. Any events containing an electron with $p_{T}$ larger than $10 \mathrm{GeV}$ are also vetoed. We additionally require $\mathbb{E}_{T}>250 \mathrm{GeV}$ and demand exactly one pair of SF/OS muon candidates with each of these muons having $p_{T}>5 \mathrm{GeV}$.

- Basic requirements on $\mu_{\mathrm{col}}$ : First we define an object $\mu_{\text {col }}$ as a two-muon system satisfying $1 \mathrm{GeV}<m_{\mu \bar{\mu}}<5 \mathrm{GeV}$ and $0.1<\Delta R_{\mu \bar{\mu}}<0.5$. Here the cut at the lower end of $\Delta R_{\mu \bar{\mu}}$ is to remove the main backgrounds where two muons emerge from a $\gamma^{*}$. The $\mu_{\text {col }}$ is required to be isolated with $I_{\text {sum }}<3 \mathrm{GeV}$. The $p_{T}$ of the $\mu_{\text {col }}$ in our signal usually tends to be small. We therefore apply a cut $p_{T}<20 \mathrm{GeV}$ for the $\mu_{\mathrm{col}}$.

- Cut on $\Delta \phi\left(\mu_{\mathrm{col}}, \mathbb{E}_{T}\right)$ : To further remove the backgrounds, we add a cut on the $\Delta \phi$ between $\mu_{\mathrm{col}}$ and $\mathbb{E}_{T}$ as $0.1<\Delta \phi<\pi / 3$. In figure 6 we show the $\Delta \phi\left(\mu_{\mathrm{col}}, \mathbb{E}_{T}\right)$ distributions for the signal corresponding to our BP2 as well as the backgrounds. We note that imposing the lower cut of $\Delta \phi>0.1$ removes much of the $\tau \bar{\tau}+j e t s$ background and the upper cut leaves only about a third of the $V+\gamma^{*}+j e t s$ background. As for the signal, this cut only removes less than $30 \%$ of the events.

- Mass cut on $\mu_{\mathrm{col}}$ : To suit the mass of the $\mu_{\mathrm{col}}$ in our signal, we only select events with $1.5 \mathrm{GeV}<m_{\mu_{\mathrm{col}}}<4 \mathrm{GeV}$, cutting off also the small window, $3.0 \mathrm{GeV}<m_{\mu_{\mathrm{col}}}<$ $3.2 \mathrm{GeV}$, corresponding to the mass of the $J / \Psi$ resonance.

- Kinematic cuts: Since in our signal the missing energy originates from two neutralinos, whereas the $\mathbb{E}_{T}$ in the $V+\gamma^{*}+$ jets background comes from a single $V$ boson, we expect different transverse mass, $M_{T}$, distributions of the signal and the backgrounds [82]. In addition, we also impose a cut on $\mathbb{E}_{T} / p_{T}\left(\mu_{\text {col }}\right)$ because the cut on the $p_{T}$ of the leading jet also results in a boosted $\mu_{\mathrm{col}}$. We can use $\mathbb{E}_{T}$ in this cut instead of the leading jet $p_{T}$ due to the comparatively much smaller $p_{T}\left(\mu_{\mathrm{col}}\right)$. In figure 7 we show the distributions of these two variables for the BP2 signal (left) and the $V+\gamma^{*}+j$ ets background (right). We find that an upper cut of $M_{T}<50 \mathrm{GeV}$ and a lower cut of $\mathbb{E}_{T} / p_{T}\left(\mu_{\mathrm{col}}\right)>20$ suppresses the background while allowing most of our signal events.

\section{Results of the signal-to-background analysis}

In table 2 we show the cut-flow for our numerical simulations of the three BPs. We note here that while generating our signal process for each BP we also required $p_{T}(\mu)>4 \mathrm{GeV}$ and $1 \mathrm{GeV}<m_{\mu \bar{\mu}}<5 \mathrm{GeV}$ at the parton level. The table shows that the total background cross section after the mono-jet cut is around $23 \mathrm{fb}$, which is three orders of magnitude larger than our signal. We observe that the $V+\gamma^{*}+$ jets background is much larger in our case than in [53]. The reason for the strong suppression of this background in [53] is that the two muons coming from the $\gamma^{*}$ are individually isolated there. However, in 

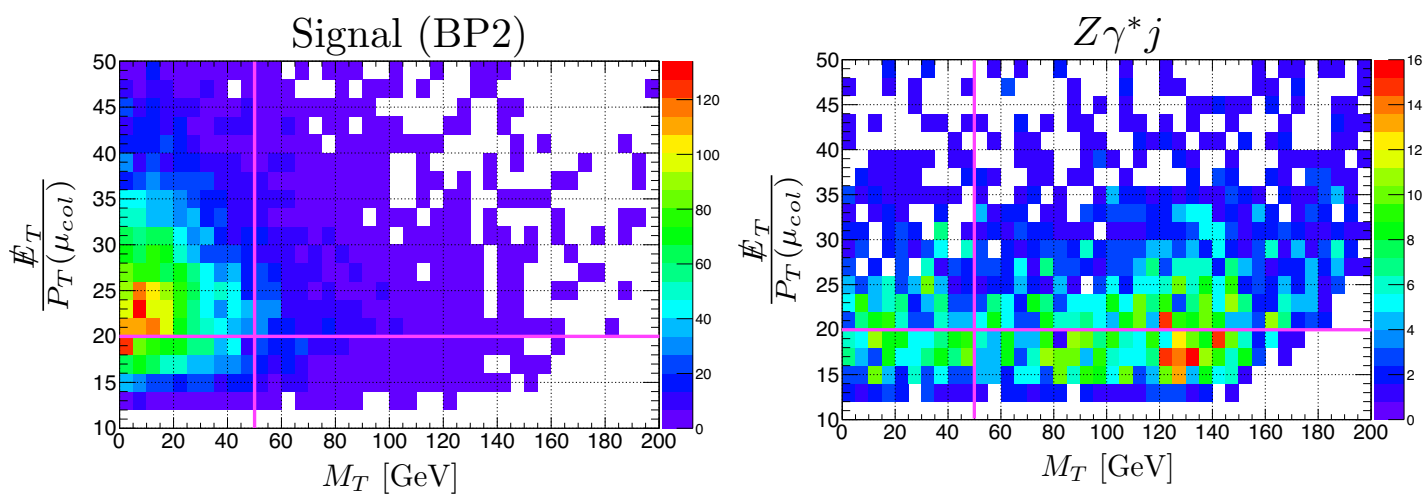

Figure 7. $M_{T}$ vs $\mathbb{E}_{T} / p_{T}\left(\mu_{\text {col }}\right)$ for the signal corresponding to our BP2 (left) and for the $Z \gamma^{*}$ background (right), after applying the mono-jet cut as well as the basic cuts on $\mu_{\mathrm{col}}$. We mark the cuts on $M_{T}$ and $\mathbb{E}_{T} / p_{T}\left(\mu_{\text {col }}\right)$ with solid magenta lines. Thus the events allowed after the cuts are the ones located in the boxes traced by the lines in the upper-left corners of the figures. The heat map corresponds to the number of events.

\begin{tabular}{|c|c|c|c|c|c|c|c|}
\hline Cuts & $W \gamma^{*} j$ & $Z \gamma^{*} j$ & $j \tau \tau$ & Total BKG & $\mathrm{BP} 1$ & BP2 & BP3 \\
\hline Mono-jet & 8.057 & 8.82 & 6.674 & 23.0 & 0.052 & 0.072 & 0.056 \\
\hline Basic $\mu_{\text {col }}$ & 0.753 & 1.05 & 0.314 & 2.1 & 0.041 & 0.042 & 0.028 \\
\hline$\Delta \phi\left(\mu_{\mathrm{col}}, \mathbb{E}_{T}\right)$ & 0.288 & 0.324 & 0.035 & 0.65 & 0.028 & 0.030 & 0.020 \\
\hline$m_{\mu_{\mathrm{col}}}$ & 0.106 & 0.118 & 0.024 & 0.248 & 0.017 & 0.023 & 0.015 \\
\hline$M_{T} \& \frac{E_{T}}{p_{T}\left(\mu_{\mathrm{col}}\right)}$ & 0.037 & 0.044 & 0.011 & 0.092 & 0.013 & 0.016 & 0.010 \\
\hline \multicolumn{5}{|c|}{$\mathrm{S} / \mathrm{B}$} & 0.14 & 0.17 & 0.11 \\
\hline \multicolumn{5}{|c|}{$S / \sqrt{B}(\sigma)$} & 2.4 & 2.9 & 1.85 \\
\hline
\end{tabular}

Table 2. Cut-flow for our signal and background processes. The cross sections are in $\mathrm{fb}$ and $S / B$ and $S / \sqrt{B}$ given in the last two rows correspond to $\mathcal{L}=3000 \mathrm{fb}^{-1}$ at the $14 \mathrm{TeV}$ LHC.

our case such an isolation condition will conversely suppress the signal process due to a comparatively smaller mass-splitting between the two higgsinos.

According to table 2, after applying all the cuts, the $V+\gamma^{*}+$ jets background is clearly the largest one. The $j \tau \bar{\tau}$ background only contributes about $10 \%$ to the total since, as noted earlier, the lower cut on $\Delta \phi\left(\mu_{\mathrm{col}}, \mathbb{E}_{T}\right)$ reduces this background by nearly an order of magnitude.

We also note in the table that the highest significance we obtained for the $14 \mathrm{TeV}$ LHC with $\mathcal{L}=3000 \mathrm{fb}^{-1}$ is $\sim 3 \sigma$ and corresponds to our BP2, while $S / B$ for this point is $17 \%$. We point out here that although both $\mathrm{BP} 1$ and BP3 give signal cross sections similar to the one obtained for BP2, the obtained significance is smallest for BP3. The reason is that for BP3 $\Delta m=5 \mathrm{GeV}$, so that $p_{T}\left(\mu_{\text {col }}\right)$ tends to be a little larger than our chosen strong upper cut on $p_{T}\left(\mu_{\mathrm{col}}\right)$. Another factor is the $m_{\mu_{\mathrm{col}}}<4 \mathrm{GeV}$ cut, which also removes some signal events in the case of BP3, but not in the case of BP2. Although we can relax the upper cut on $p_{T}\left(\mu_{\mathrm{col}}\right)$ to around $25 \mathrm{GeV}$, it will also result in larger backgrounds and 
thus the statistical significance for BP3 will not improve. We should also point out here that for BP1, where $\Delta m=3 \mathrm{GeV}$, since $m_{\mu_{\mathrm{col}}}$ should be less than $3 \mathrm{GeV}$, changing the upper cut on $m_{\mu_{\mathrm{col}}}$ from $4 \mathrm{GeV}$ to $3 \mathrm{GeV}$ would enhance our signal significance. However, in the experimental searches the true value of the higgsino mass-splitting is unknown. We therefore retain the upper cut of $4 \mathrm{GeV}$, which suits most of the $\Delta m$ range that we are interested in.

Finally, using our method the minimum $\Delta m$ we have managed to explore is $\sim 3 \mathrm{GeV}$. This is because of the requirement of $p_{T}>5 \mathrm{GeV}$ for the muons. By imposing a lower cut, $p_{T}>4 \mathrm{GeV}$ [83], for the muons, a good sensitivity to even smaller values of $\Delta m$ can be achieved.

\section{Conclusions}

In this article, we have analyzed the possibility of probing natural SUSY scenarios with a highly compressed higgsino mass spectrum at the $14 \mathrm{TeV}$ LHC, using the collinearity between the two muons produced in such scenarios. We have found that a statistical significance of up to $3 \sigma$ as well as $S / B$ up to $17 \%$ can be obtained for $\Delta m=m_{\tilde{\chi}_{2}^{0}}-m_{\tilde{\chi}_{1}^{0}}=$ $4 \mathrm{GeV}$ and $\mathrm{a} \sim 120 \mathrm{GeV} \tilde{\chi}_{1}^{0}$ with an integrated luminosity of $3000 \mathrm{fb}^{-1}$ at the LHC. In fact, by using our analysis method but further lowering the cut we imposed on the $p_{T}$ of muons, MSSM parameter space regions with $\Delta m$ even lower than $3 \mathrm{GeV}$ can be explored.

\section{Acknowledgments}

This work is supported by the Korea Ministry of Science, ICT and Future Planning, Gyeongsangbuk-Do and Pohang City for Independent Junior Research Groups at the Asia Pacific Center for Theoretical Physics. MP is also supported by World Premier International Research Center Initiative (WPI Initiative), MEXT, Japan. CCH thanks the Kavli IPMU for its warm hospitality and appreciates encouragement by Hitoshi Murayama.

Open Access. This article is distributed under the terms of the Creative Commons Attribution License (CC-BY 4.0), which permits any use, distribution and reproduction in any medium, provided the original author(s) and source are credited.

\section{References}

[1] ATLAS collaboration, Observation of a new particle in the search for the Standard Model Higgs boson with the ATLAS detector at the LHC, Phys. Lett. B 716 (2012) 1 [arXiv: 1207.7214] [INSPIRE].

[2] CMS collaboration, Observation of a new boson at a mass of $125 \mathrm{GeV}$ with the CMS experiment at the LHC, Phys. Lett. B 716 (2012) 30 [arXiv:1207.7235] [INSPIRE].

[3] M. Carena and H.E. Haber, Higgs boson theory and phenomenology, Prog. Part. Nucl. Phys. 50 (2003) 63 [hep-ph/0208209] [INSPIRE]. 
[4] A. Arbey, M. Battaglia, A. Djouadi, F. Mahmoudi and J. Quevillon, Implications of a 125 GeV Higgs for supersymmetric models, Phys. Lett. B 708 (2012) 162 [arXiv:1112.3028] [INSPIRE].

[5] M. Carena, S. Gori, N.R. Shah and C.E.M. Wagner, A 125 GeV SM-like Higgs in the MSSM and the $\gamma \gamma$ rate, JHEP 03 (2012) 014 [arXiv: 1112.3336] [INSPIRE].

[6] J. Cao, Z. Heng, D. Li and J.M. Yang, Current experimental constraints on the lightest Higgs boson mass in the constrained MSSM, Phys. Lett. B 710 (2012) 665 [arXiv:1112.4391] [INSPIRE].

[7] J.-J. Cao, Z.-X. Heng, J.M. Yang, Y.-M. Zhang and J.-Y. Zhu, A SM-like Higgs near 125 GeV in low energy SUSY: a comparative study for MSSM and NMSSM, JHEP 03 (2012) 086 [arXiv: 1202.5821] [INSPIRE].

[8] J. Cao, Z. Heng, J.M. Yang and J. Zhu, Status of low energy SUSY models confronted with the LHC $125 \mathrm{GeV}$ Higgs data, JHEP 10 (2012) 079 [arXiv:1207.3698] [INSPIRE].

[9] CMS collaboration, Search for supersymmetry using razor variables in events with b-jets in pp collisions at $8 \mathrm{TeV}$, Phys. Rev. D 91 (2015) 052018 arXiv: 1502.00300 [InSPIRE].

[10] CMS collaboration, Search for Supersymmetry in pp collisions at $8 \mathrm{TeV}$ in events with a single lepton, multiple jets and b-tags, CMS-PAS-SUS-13-007.

[11] ATLAS collaboration, Search for new phenomena using final states with large jet multiplicities and missing transverse momentum with ATLAS in $20 \mathrm{fb}^{-1}$ of $\sqrt{\mathrm{s}}=8 \mathrm{TeV}$ proton-proton collisions, ATLAS-CONF-2013-054, ATLAS-COM-CONF-2013-060.

[12] ATLAS collaboration, Search for strong production of supersymmetric particles in final states with missing transverse momentum and at least three b-jets using $20.1 \mathrm{fb}^{-1}$ of pp collisions at sqrt $(s)=8 \mathrm{TeV}$ with the ATLAS Detector, JHEP 10 (2014) 024 [arXiv:1407.0600] [INSPIRE].

[13] D.M. Ghilencea, H.M. Lee and M. Park, Tuning supersymmetric models at the LHC: A comparative analysis at two-loop level, JHEP 07 (2012) 046 [arXiv:1203.0569] [INSPIRE].

[14] R.L. Arnowitt and P. Nath, Loop corrections to radiative breaking of electroweak symmetry in supersymmetry, Phys. Rev. D 46 (1992) 3981 [INSPIRE].

[15] S.P. Martin, Non-universal gaugino masses from non-singlet F-terms in non-minimal unified models, Phys. Rev. D 79 (2009) 095019 [arXiv:0903.3568] [InSPIRE].

[16] J.E. Younkin and S.P. Martin, Non-universal gaugino masses, the supersymmetric little hierarchy problem and dark matter, Phys. Rev. D 85 (2012) 055028 [arXiv:1201.2989] [INSPIRE].

[17] S. Akula and P. Nath, Gluino-driven radiative breaking, Higgs boson mass, muon g-2 and the Higgs diphoton decay in supergravity unification, Phys. Rev. D 87 (2013) 115022 [arXiv: 1304.5526] [INSPIRE].

[18] I. Gogoladze, F. Nasir and Q. Shafi, Non-Universal Gaugino Masses and Natural Supersymmetry, Int. J. Mod. Phys. A 28 (2013) 1350046 [arXiv:1212.2593] [inSPIRE].

[19] C.H. Chen, M. Drees and J.F. Gunion, A Nonstandard string/SUSY scenario and its phenomenological implications, Phys. Rev. D 55 (1997) 330 [Erratum ibid. D 60 (1999) 039901] [hep-ph/9607421] [INSPIRE]. 
[20] J.F. Gunion and S. Mrenna, A Study of SUSY signatures at the Tevatron in models with near mass degeneracy of the lightest chargino and neutralino, Phys. Rev. D 62 (2000) 015002 [hep-ph/9906270] [INSPIRE].

[21] C.H. Chen, M. Drees and J.F. Gunion, Searching for invisible and almost invisible particles at $e^{+} e^{-}$colliders, Phys. Rev. Lett. 76 (1996) 2002 [hep-ph/9512230] [INSPIRE].

[22] Q.-H. Cao, C.-R. Chen, C.S. Li and H. Zhang, Effective Dark Matter Model: Relic density, CDMS II, Fermi LAT and LHC, JHEP 08 (2011) 018 [arXiv:0912.4511] [InSPIRE].

[23] M. Beltrán, D. Hooper, E.W. Kolb, Z.A.C. Krusberg and T.M.P. Tait, Maverick dark matter at colliders, JHEP 09 (2010) 037 [arXiv: 1002.4137] [INSPIRE].

[24] G.F. Giudice, T. Han, K. Wang and L.-T. Wang, Nearly Degenerate Gauginos and Dark Matter at the LHC, Phys. Rev. D 81 (2010) 115011 [arXiv:1004.4902] [INSPIRE].

[25] J. Goodman et al., Constraints on Dark Matter from Colliders, Phys. Rev. D 82 (2010) 116010 [arXiv: 1008.1783] [INSPIRE].

[26] A. Rajaraman, W. Shepherd, T.M.P. Tait and A.M. Wijangco, LHC Bounds on Interactions of Dark Matter, Phys. Rev. D 84 (2011) 095013 [arXiv:1108.1196] [inSPIRE].

[27] P.J. Fox, R. Harnik, J. Kopp and Y. Tsai, Missing Energy Signatures of Dark Matter at the LHC, Phys. Rev. D 85 (2012) 056011 [arXiv:1109.4398] [inSPIRE].

[28] C. Han et al., Probing Light Higgsinos in Natural SUSY from Monojet Signals at the LHC, JHEP 02 (2014) 049 [arXiv: 1310.4274] [INSPIRE].

[29] P. Schwaller and J. Zurita, Compressed electroweakino spectra at the LHC, JHEP 03 (2014) 060 [arXiv:1312.7350] [INSPIRE].

[30] ATLAS collaboration, Search for dark matter candidates and large extra dimensions in events with a jet and missing transverse momentum with the ATLAS detector, JHEP 04 (2013) 075 [arXiv: 1210.4491] [INSPIRE].

[31] CMS collaboration, Search for dark matter and large extra dimensions in monojet events in pp collisions at $\sqrt{s}=7 \mathrm{TeV}$, JHEP 09 (2012) 094 [arXiv: 1206.5663] [INSPIRE].

[32] CMS collaboration, Search for dark matter, extra dimensions and unparticles in monojet events in proton-proton collisions at $\sqrt{s}=8 \mathrm{TeV}$, arXiv:1408.3583 [INSPIRE].

[33] H. Baer, A. Mustafayev and X. Tata, Monojets and mono-photons from light higgsino pair production at LHC14, Phys. Rev. D 89 (2014) 055007 [arXiv:1401.1162] [INSPIRE].

[34] G. Brooijmans et al., Les Houches 2013: Physics at TeV Colliders: New Physics Working Group Report, arXiv: 1405.1617 [InSPIRE].

[35] A. Anandakrishnan, L.M. Carpenter and S. Raby, Degenerate gaugino mass region and mono-boson collider signatures, Phys. Rev. D 90 (2014) 055004 [arXiv:1407.1833] [INSPIRE].

[36] S. Gori, S. Jung, L.-T. Wang and J.D. Wells, Prospects for Electroweakino Discovery at a $100 \mathrm{TeV}$ Hadron Collider, JHEP 12 (2014) 108 [arXiv:1410.6287] [INSPIRE].

[37] K. Rolbiecki and K. Sakurai, Constraining compressed supersymmetry using leptonic signatures, JHEP 10 (2012) 071 [arXiv:1206.6767] [INSPIRE].

[38] S. Gori, S. Jung and L.-T. Wang, Cornering electroweakinos at the LHC, JHEP 10 (2013) 191 [arXiv: 1307.5952] [INSPIRE]. 
[39] M. Berggren et al., Electroweakino Searches: A Comparative Study for LHC and ILC (A Snowmass White Paper), arXiv:1309.7342 [INSPIRE].

[40] T. Han, S. Padhi and S. Su, Electroweakinos in the Light of the Higgs Boson, Phys. Rev. D 88 (2013) 115010 [arXiv:1309.5966] [INSPIRE].

[41] K.-i. Hikasa, T. Liu, L. Wang and J.M. Yang, Pseudo-goldstino and electroweak gauginos at the LHC, JHEP 07 (2014) 065 [arXiv: 1403.5731] [INSPIRE].

[42] T. Han, Z. Liu and S. Su, Light Neutralino Dark Matter: Direct/Indirect Detection and Collider Searches, JHEP 08 (2014) 093 [arXiv:1406.1181] [INSPIRE].

[43] G. Barenboim, E.J. Chun, S. Jung and W.I. Park, Implications of an axino LSP for naturalness, Phys. Rev. D 90 (2014) 035020 [arXiv:1407.1218] [INSPIRE].

[44] J. Bramante, A. Delgado, F. Elahi, A. Martin and B. Ostdiek, Catching sparks from well-forged neutralinos, Phys. Rev. D 90 (2014) 095008 [arXiv: 1408.6530] [inSPIRE].

[45] C. Han, L. Wu, J.M. Yang, M. Zhang and Y. Zhang, New approach for detecting a compressed bino/wino at the LHC, Phys. Rev. D 91 (2015) 055030 [arXiv:1409.4533] [INSPIRE].

[46] T.A.W. Martin and D. Morrissey, Electroweakino constraints from LHC data, JHEP 12 (2014) 168 [arXiv:1409.6322] [INSPIRE].

[47] C. Han, Probing light bino and higgsinos at the LHC, arXiv:1409.7000 [INSPIRE].

[48] T. Liu, L. Wang and J.M. Yang, Pseudo-goldstino and electroweakinos via VBF processes at LHC, JHEP 02 (2015) 177 [arXiv: 1411.6105] [INSPIRE].

[49] Z. Han and Y. Liu, MT2 to the Rescue - Searching for Sleptons in Compressed Spectra at the LHC, arXiv:1412.0618 [INSPIRE].

[50] J. Bramante et al., Relic neutralino surface at a $100 \mathrm{TeV}$ collider, Phys. Rev. D 91 (2015) 054015 [arXiv: 1412 .4789] [INSPIRE].

[51] A. Barr and J. Scoville, A boost for the EW SUSY hunt: monojet-like search for compressed sleptons at LHC14 with $100 \mathrm{fb}^{-1}$, arXiv:1501.02511 [INSPIRE].

[52] Z. Han, G.D. Kribs, A. Martin and A. Menon, Hunting quasidegenerate Higgsinos, Phys. Rev. D 89 (2014) 075007 [arXiv:1401.1235] [INSPIRE].

[53] H. Baer, A. Mustafayev and X. Tata, Monojet plus soft dilepton signal from light higgsino pair production at LHC14, Phys. Rev. D 90 (2014) 115007 [arXiv:1409.7058] [INSPIRE].

[54] C. Brust, A. Katz, S. Lawrence and R. Sundrum, SUSY, the Third Generation and the LHC, JHEP 03 (2012) 103 [arXiv:1110.6670] [INSPIRE].

[55] M. Papucci, J.T. Ruderman and A. Weiler, Natural SUSY Endures, JHEP 09 (2012) 035 [arXiv:1110.6926] [INSPIRE].

[56] L.J. Hall, D. Pinner and J.T. Ruderman, A Natural SUSY Higgs Near 126 GeV, JHEP 04 (2012) 131 [arXiv:1112.2703] [INSPIRE].

[57] J.L. Feng and D. Sanford, A Natural 125 GeV Higgs Boson in the MSSM from Focus Point Supersymmetry with A-Terms, Phys. Rev. D 86 (2012) 055015 [arXiv:1205.2372] [INSPIRE].

[58] J. Cao, C. Han, L. Wu, J.M. Yang and Y. Zhang, Probing Natural SUSY from Stop Pair Production at the LHC, JHEP 11 (2012) 039 [arXiv:1206.3865] [InSPIRE]. 
[59] H. Baer, V. Barger, P. Huang, A. Mustafayev and X. Tata, Radiative natural SUSY with a 125 GeV Higgs boson, Phys. Rev. Lett. 109 (2012) 161802 [arXiv:1207.3343] [InSPIRE].

[60] C. Han, F. Wang and J.M. Yang, Natural SUSY from SU(5) Orbifold GUT, JHEP 11 (2013) 197 [arXiv: 1304.5724] [INSPIRE].

[61] C. Han, K.-i. Hikasa, L. Wu, J.M. Yang and Y. Zhang, Current experimental bounds on stop mass in natural SUSY, JHEP 10 (2013) 216 [arXiv:1308.5307] [INSPIRE].

[62] K. Kowalska and E.M. Sessolo, Natural MSSM after the LHC 8 TeV run, Phys. Rev. D 88 (2013) 075001 [arXiv:1307.5790] [INSPIRE].

[63] A. Djouadi, M.M. Muhlleitner and M. Spira, Decays of supersymmetric particles: The Program SUSY-HIT (SUspect-SdecaY-HDECAY-InTerface), Acta Phys. Polon. B 38 (2007) 635 [hep-ph/0609292] [INSPIRE].

[64] XENON1T collaboration, E. Aprile, The XENON1T Dark Matter Search Experiment, Springer Proc. Phys. C12-02-22 (2013) 93 [arXiv:1206.6288] [INSPIRE].

[65] CMS collaboration, Search for disappearing tracks in proton-proton collisions at $\sqrt{s}=8$ TeV, JHEP 01 (2015) 096 [arXiv:1411.6006] [INSPIRE].

[66] N. Arkani-Hamed and N. Weiner, LHC Signals for a SuperUnified Theory of Dark Matter, JHEP 12 (2008) 104 [arXiv:0810.0714] [INSPIRE].

[67] N. Arkani-Hamed, D.P. Finkbeiner, T.R. Slatyer and N. Weiner, A Theory of Dark Matter, Phys. Rev. D 79 (2009) 015014 [arXiv:0810.0713] [InSPIRE].

[68] M. Baumgart, C. Cheung, J.T. Ruderman, L.-T. Wang and I. Yavin, Non-Abelian Dark Sectors and Their Collider Signatures, JHEP 04 (2009) 014 [arXiv: 0901.0283] [InSPIRE].

[69] A. Katz and R. Sundrum, Breaking the Dark Force, JHEP 06 (2009) 003 [arXiv:0902.3271] [INSPIRE].

[70] C. Cheung, J.T. Ruderman, L.-T. Wang and I. Yavin, Lepton Jets in (Supersymmetric) Electroweak Processes, JHEP 04 (2010) 116 [arXiv:0909.0290] [INSPIRE].

[71] A. Falkowski, J.T. Ruderman, T. Volansky and J. Zupan, Hidden Higgs Decaying to Lepton Jets, JHEP 05 (2010) 077 [arXiv: 1002.2952] [INSPIRE].

[72] CMS collaboration, Search for a non-standard-model Higgs boson decaying to a pair of new light bosons in four-muon final states, Phys. Lett. B 726 (2013) 564 [arXiv:1210.7619] [INSPIRE].

[73] CMS collaboration, Search for Light Resonances Decaying into Pairs of Muons as a Signal of New Physics, JHEP 07 (2011) 098 [arXiv:1106.2375] [INSPIRE].

[74] J. Alwall et al., The automated computation of tree-level and next-to-leading order differential cross sections and their matching to parton shower simulations, JHEP 07 (2014) 079 [arXiv: 1405.0301] [INSPIRE].

[75] T. Sjöstrand, S. Mrenna and P.Z. Skands, PYTHIA 6.4 Physics and Manual, JHEP 05 (2006) 026 [hep-ph/0603175] [inSPIRE].

[76] DELPHES 3 collaboration, J. de Favereau et al., DELPHES 3, A modular framework for fast simulation of a generic collider experiment, JHEP 02 (2014) 057 [arXiv:1307.6346] [INSPIRE].

[77] M. Cacciari, G.P. Salam and G. Soyez, FastJet User Manual, Eur. Phys. J. C 72 (2012) 1896 [arXiv: 1111.6097] [INSPIRE]. 
[78] M. Cacciari, G.P. Salam and G. Soyez, The Anti-k(t) jet clustering algorithm, JHEP 04 (2008) 063 [arXiv:0802.1189] [INSPIRE].

[79] A. Denner, S. Dittmaier, T. Kasprzik and A. Mück, Electroweak corrections to monojet production at the LHC, Eur. Phys. J. C 73 (2013) 2297 [arXiv:1211.5078] [INSPIRE].

[80] F. Caravaglios, M.L. Mangano, M. Moretti and R. Pittau, A New approach to multijet calculations in hadron collisions, Nucl. Phys. B 539 (1999) 215 [hep-ph/9807570] [InSPIRE].

[81] ATLAS collaboration, Search for pair-produced third-generation squarks decaying via charm quarks or in compressed supersymmetric scenarios in pp collisions at $\sqrt{s}=8 \mathrm{TeV}$ with the ATLAS detector, Phys. Rev. D 90 (2014) 052008 [arXiv:1407.0608] [INSPIRE].

[82] G.F. Giudice, B. Gripaios and R. Mahbubani, Counting dark matter particles in LHC events, Phys. Rev. D 85 (2012) 075019 [arXiv:1108.1800] [INSPIRE].

[83] ATLAS collaboration, A search for prompt lepton-jets in pp collisions at $\sqrt{s}=7 \mathrm{TeV}$ with the ATLAS detector, Phys. Lett. B 719 (2013) 299 [arXiv:1212.5409] [InSPIRE]. 